\title{
Pulsed dose rate brachytherapy - is it the right way?
}

\author{
Janusz Skowronek, MD, PhD, Ass. Prof. \\ Brachytherapy Department, Greater Poland Cancer Centre, Poznan, Poland
}

\begin{abstract}
Pulsed dose rate (PDR-BT) treatment is a brachytherapy modality that combines physical advantages of high-doserate (HDR-BT) technology (isodose optimization, radiation safety) with the radiobiological advantages of low-dose-rate (LDR-BT) brachytherapy. Pulsed brachytherapy consists of using stronger radiation source than for LDR-BT and producing series of short exposures of 10 to 30 minutes in every hour to approximately the same total dose in the same overall time as with the LDR-BT. Modern afterloading equipment offers certain advantages over interstitial or intracavitary insertion of separate needles, tubes, seeds or wires. Isodose volumes in tissues can be created flexibly by a combination of careful placement of the catheter and the adjustment of the dwell times of the computerized stepping source. Automatic removal of the radiation sources into a shielded safe eliminates radiation exposures to staff and visitors. Radiation exposure is also eliminated to the staff who formerly loaded and unloaded multiplicity of radioactive sources into the catheters, ovoids, tubes etc. This review based on summarized clinical investigations, analyses the feasibility and the background to introduce this brachytherapy technique and chosen clinical applications of PDR-BT.
\end{abstract}

J Contemp Brachyther 2010; 2, 3: 107-113 DOI: 10.5114/jcb.2010.16921

Key words: indications, PDR brachytherapy, pulsed dose, radiobiology.

\section{Purpose}

The efficacy of brachytherapy is attributed to the ability of radioactive implants to deliver higher concentrated radiation dose more precisely to the tissues than external beam radiation therapy (EBRT) alone. This contributes to improve local control, providing clinical delimitation and access to the tissues as well as better protection of surrounding healthy tissues. In contrast to EBRT, brachytherapy is quite invasive and requires an insertion of site-specific applicators under sedation or anesthesia. The surgeon is occasionally involved in these procedures, particularly if laparotomy or craniotomy is necessary for insertion of the applicators or if tumor resection is required prior to applicator insertion. The specialist should always be aware of the indications for brachytherapy and the associated techniques [1-3]. Brachytherapy with modern afterloading equipment offers three major advantages over interstitial or intracavitary insertion of separate needles, tubes, seeds or wires: 1) isodose volumes in tissues can be created flexibly by a combination of careful placement of the catheter and adjustment of the dwell times of computerized stepping source; this process is usually called "dose optimization", 2) automatic removal of the radiation sources into a shielded safe whenever somebody enters the procedure room eliminates radiation exposures to staff and visitors, 3) radiation exposure is also eliminated to the staff who formerly loaded and unloaded multiplicity of radioactive sources into the catheters, ovoids, tubes, etc. [1,4-7].
LDR-BT remote afterloading systems certainly offer radiation protection, but do not provide so much of flexibility in order to design an alternative isodose volumes as higher dose rate sources with adjustable stepping positions and dwell times. At the other end of the spectrum is the use of HDR-BT afterloading with a single source of $10 \mathrm{Ci}$ 192Ir moved by a computer into a series of dwell positions, so that the choice of isodose volume is very flexible. Large doses can be applied within a few minutes. Such sources require well-shielded bunkers that are similar to linear accelerator room. There is a radiobiological disadvantage in using such high dose rates of 1-3 Gy/min (greater ratio of late tissue effects), which in practice can be overcome by a careful placement of the catheters and by good immobility achievable with very short exposures. PDR-BT is a brachytherapy modality that combines physical advantages of high-dose-rate (HDR-BT) technology (isodose optimization, planning flexibility, radiation safety) with the radiobiological advantages of low-doserate (LDR-BT) brachytherapy (repair advantages).

\section{Method description}

PDR-BT uses a single stepping source of 15-37 GBq (0,5-1 Ci) of 192Iridium. This produces treatment dose rates of up to 3 Gy per hour which can be utilized (pulsed) each hour (most frequently), 24 pulses per day. The source is enclosed in a capsule of $1.1 \mathrm{~mm}$ diameter and $2.5 \mathrm{~mm}$ length. The single radioactive stepping source moves through all implanted catheters during each pulse. 
PDR-BT consists of using stronger radiation source than for LDR-BT and producing a series of short exposures of 10 to 30 minutes in every hour to approximately the same total dose in the same overall as with the LDR-BT. Trajectory through the implanted catheter of a single high activity source can be precisely programmed by a dedicated computer and carried out by a remote source projector. The resulting isodoses may be optimized by modulating the dwell time of the source as a function of its trajectory within the implanted volume. The optimization allows individualization of dose distributions, while essentially eliminating radiation exposure to the medical staff. The source strength is 10 to 20 times lower than of HDR-BT, where requirements for shielding are less stringent. An average brachytherapy room would require less than two extra-half value thickness of protection, if any; an accelerator type bunker is not necessary. Nursing care is facilitated compared with LDR-BT, since patients can be attended between the treatment sessions without concerning about unnecessary radiation exposure.

\section{Radiobiology of PDR-BT}

The gap between the pulses allows greater freedom for the patient and increased safety of the nursing staff. In principle, any move away from continuous exposure towards treatment with gaps, carries a radiobiological disadvantage. This is equivalent to fractionation with a larger dose per fraction and theoretical and experimental evidence that this could lead to a relative increase in late normal-tissue reactions is strong. The magnitude of this effect has been considered by Brenner and Hall who concluded that for gaps between pulses of up to 60 minutes the radiobiological deficit may be acceptable [8]. In PDR-BT each pulse delivers a small dose and is followed by an interval which allows some repair, therefore the increase of radiobiological effect should be small. However, the main question is whether or not the increased effect is greater on late-responding normal tissues than on tumor cell kill.

To reproduce the biological effects of LDR-BT using PDR remote afterloading Brenner and Hall [8] and Fowler and Mount [9] give the following four recommendations: 1) the same total dose, 2) the same dose rate: typically about $0.5 \mathrm{~Gy} /$ hour, 3) pulse length of 10 minutes or more (or dose rate not exceeding $3 \mathrm{~Gy} /$ hour during the pulse), 4) each hour pulse repetition: typically $0.4-1.0 \mathrm{~Gy} /$ hour. If these conditions are met, the biological effects of PDR radiation therapy should be equivalent to those of LDRBT for all tissues. These conclusions were prepared based on calculations, taking into account cell repair capacity (estimated by $\alpha / \beta$ ) and the kinetics of the repair (estimated by $\mathrm{T}_{1 / 2}$ ), for both tumors and late-reacting normal tissues. The value of $\alpha / \beta$ for tumors and late reacting human tissues have been estimated and are consistent with laboratory results using experimental animals. By contrast, because of a lack of clinical data, $\mathrm{T}_{1 / 2}$ has been estimated from experimental data [10]. However, it is likely that early-responding tissues such as tumors do repair sublethal damage more rapidly than the late-responding tissues. In 1996, Brenner and Hall exploited this difference to design new therapeutic regimens. They estimated, using a $\mathrm{T}_{1 / 2}$ of
0.5 hours for early-responding tissues and 4 hours for lateresponding, that PDR-BT delivering series of pulses separated by 3-4 hours should produce better results than LDR-BT [11-13]. Advantages of PDR-BT include: 1) full radiation protection, 2) no source preparation, 3) no source inventory, 4) optimization of the dose rate distribution, 5) only one source to be replaced every three months, 6) all brachytherapy methods are feasible with one machine: intracavitary, interstitial, intraoperative, intraluminal. Limitations of PDR-BT include: 1) only one person per day can by treated, 2) another disadvantage of the system compared with LDR-BT is the presence of connecting tubes between the machine and the needles (catheters), the weight of the system which may cause some discomfort, 3) the multiple source transfers may result in treatment irregularities due to source blockages, particularly in case of implanted plastic tubes.

Although the PDR-BT approach has been the subject of numerous theoretical papers, and afterloading machines modified for PDR-BT have been commercially available for several years, insufficient number of papers has been published regarding clinical experience with these techniques [14-16]. Some of them are discussed below.

\section{Clinical investigations}

\section{Gynecological tumors}

De Pree et al. [4] described PDR-BT results of 16 gynecological patients ( 8 patients with primary cervical cancer, 1 case of recurrent cervical cancer, 5 vaginal cancer, 2 patients with recurrent endometrial cancer with vaginal infiltration). Pulse dose ranged from 0.4 to $1 \mathrm{~Gy}$, median total dose - 20 Gy, specified in CTV (clinical target volume) similarly to EBRT. Overall free survival (OFS) rate was $43.7 \%$ (7 patients) with median follow-up of 18 months. Observed complications were: radiation toxicity in vagina with fistula $(n=2)$, dysuria $(n=3)$, nocturia $(n=2)$, diarrhea $(n=3)$, temporary pain during treatment. Klimek et al. [17] discussed adjuvant therapy after hysterectomy in 110 patients with endometrial cancer. Median total PDR-BT dose was $21 \mathrm{~Gy}, 67$ patients (61\%) additionally received EBRT. Median follow-up was 15 months and local recurrence was noted in 3 cases $(2.7 \%)$. Early radiation reactions in vagina and rectum were observed in 5 and 4 cases, respectively. Late reactions were noted: in vagina $(n=1)$, in rectum $(n=5)$, in urinary bladder $(n=2)$. In 3 cases $(2.7 \%)$ local recurrence during follow-up was observed. In another paper [2] authors presented PDR-BT results of 23 patients with cervical cancer, 6 cases with endometrial cancer and 3 with vaginal cancer. Pulse dose ranged from 0.4 to $0.8 \mathrm{~Gy} / \mathrm{h}$, total doses differed depending on EBRT dose. After one year of follow-up no failure was noted. In 3 cases early reactions were observed such as mucositis, infections and pain. Serkies et al. [18] used PDR-BT as a palliative way of treatment in 7 patients with cervical cancer and in 11 patients with endometrial cancer. PDR-BT was chosen instead of LDR-BT because of contraindications for long-term immobilization and patients negative response towards LDR-BT treatment. In 11 cases PDR-BT was combined with EBRT, in 2 - was 
used after surgery; 5 patients were treated for vaginal recurrence. Pulse dose ranged from 0.5 to $2.5 \mathrm{~Gy}$, hourly. In 4 cases, in order to shorten the total treatment time, pulse dose was increased to 4-6 Gy hourly. In 11/18 cases complete remission was observed in 6 to13 months of follow-up, 3 died due to progression in $7^{\text {th }}, 10^{\text {th }}$ and $15^{\text {th }}$ month, 2 died for other reason. Swift et al. [19] presented a group of 65 patients treated with PDR-BT, pulse dose $0.40-0.85$ Gy. Early complications rate was $6.5 \%$, late complications rate $-15 \%$ in 2 years. Long-term control was achieved in 48 out of 65 cases. Rogers et al. [21] treated 52 patients with cervical cancer using $0.55 \mathrm{~Gy} /$ pulse and combining with EBRT. Median EBRT dose was 45 Gy in 22 fractions, together with PDR-BT - 75.8 Gy (interstitial) or 84.1 Gy in point A (intracavitary). In 2 cases Grade IV complications were noted (bladder-vaginal fistula, rectumvaginal fistula), in 1 case Grade III (hematuria) and in 5 cases - Grade II. Overall free survival (OFS) rate (4 years follow-up) was $66 \%$. Jensen et al. [21] presented PDR-BT results (pulse dose $0.6 \mathrm{~Gy}$ ) of 34 patients with locally advanced gynecological tumor $(n=12)$ or recurrence $(n=22)$. The group included 25 patients with cervical cancer, 7 with endometrial cancer, 2 cases with vulvar cancer. EBRT total dose was 46 Gy/23 fractions, PDR-BT $30 \mathrm{~Gy}$, pulse dose $0.6 \mathrm{~Gy} /$ hourly. Overall survival (OS) rate was $71 \%$ and $63 \%$ (1 and 2 years, respectively) and was higher for recurrence group $(85 \%)$ then for locally advanced $-58 \%$. Authors reported this combined treatment as effective but noted relatively high early and late complications rate. In 10 patients Grade III late complications were observed. An overview of PDR-BT experience in gynecological tumors was presented in an earlier review paper by Skowronek et al. [16].

\section{Breast cancer}

PDR brachytherapy has an established place in treatment of breast cancer [22]. Fritz et al. [23] assessed the feasibility and morbidity of PDR boost after breast conserving surgery (BCS) and EBRT, with flexible breast implants. Sixty-five high risk patients were treated with interstitial PDR boost. The inclusion criteria for interstitial boost were as follows: positive or close margin after resection, extensive intraductal component (EIC), intralymphatic extension, lobular carcinoma, T2 tumors and high nuclear grade (GIII). Dose calculation and specification were performed using Paris system. The dose per pulse was 1 Gy/hourly. The treatment schedule was 50 Gy EBRT to the whole breast and $20 \mathrm{~Gy}$ boost. The median follow-up was 30 months (12-54 months). Sixty percent of the patients described their cosmetic result as excellent, $27 \%$ as good, $11 \%$ estimated as fair and $2 \%$ as poor. Eighty-six percent of the patients had no radiogenous skin changes in the boost area. In $11 \%$ of patients minimal punctiform telangiectasia appealed at single puncture sites. In $3 \%$ $(2 / 65)$ of patients planar telangiectasia appeared on the medial side of the implant. The rate of isolated local recurrences was $1.5 \%$. Authors concluded that the interstitial CLDR boost of the breast can be replaced by PDR technique without severe acute and late complications and without deterioration of cosmetic results [23].
20 patients with breast cancer were treated by Serkies et al. [18]: 16 received PDR dose as a boost after EBRT, 4 with locally advanced breast cancer (LABC) received 60 Gy with EBRT and 10 to 20 Gy with PDR-BT. Local remission was achieved in all cases. Harms et al. [24] evaluated effect, toxicity and cosmetic results of a prospectively applied PDR-BT boost schedule in patients with stage I/II/IIIa invasive breast cancer. 113 patients were treated after breast-conserving surgery (BCS) and EBRT (median dose 50 Gy, ranged 46-52). The boost dose was graded in accordance to the pathologic tumor characteristics: 20-25 Gy - incomplete resection $(n=34)$, vascular invasion $(n=27)$, close margin resection $(n=41)$; 15 Gy - T2G3 stage $(n=11)$. The overall local failure rate after a median follow-up of 61 months was $4.4 \%$ (5/113). The actuarial 5- and 8-year local recurrence-free survival rates were $95 \%$ and $93 \%$, respectively. Cosmetic effect was rated by $90 \%$ of the patients as excellent or good. 14/113 patients experienced Grade III (all caused by planar telangiectasia) and none of the patients Grade IV late toxicity of the skin (RTOG/EORTC). A boost dose of $25 \mathrm{~Gy}$ resulted in a significantly higher rate of late toxicity (Fisher's exact test, $p<0.01$ ). Authors concluded that PDR-BT brachytherapy was safe, effective and provided good cosmetic results. A CLDR breast boost can be replaced by PDR-BT without significant loss of therapeutic ratio [24]. Mangold et al. [25] analyzed quality control of PDR-BT. In the Radiotherapy Department of Leuven, about $20 \%$ of all breast cancer patients treated with BCS and EBRT received an additional boost with PDR-BT. Firstly, an investigation was performed to assess the accuracy of the delivered PDR-BT treatment. Secondly, the feasibility of in vivo measurements during PDR dose delivery was investigated. Two phantoms were manufactured to mimic a breast, one for thermoluminescent dosimetry (TLD) measurements, and one for dosimetry using radiochromic films. The dose distributions calculated with the TPS were in good agreement with both TLD and radiochromic film measurements (average deviations of point doses, $< \pm 5 \%$ ). They concluded that most of the deviations between measured and calculated doses were in the order of magnitude of uncertainty associated with the source strength specification, except for the point doses measured close to the skin. In vivo dosimetry during PDR brachytherapy treatment was found to be valuable procedure in detecting large errors, e.g. errors caused by an incorrect data transfer. Johansson et al. [26] evaluated long time outcome with regard to local tumor control, cosmetic outcome and side effects of a short ( 5 days) accelerated interstitial brachytherapy (APBI) delivered to the surroundings of the operated sector. 50 patients with early T1 and T2 breast cancer were treated with APBI. Radical sector resection was performed and followed later by an interstitial pulsed dose rate (PDR) brachytherapy of 50 Gy in 5 days. The treatment was centered on the tumor with a margin of $30 \mathrm{~mm}$. One patient was treated bilaterally. Patients were followed-up with median of 86 (32-126) months. Ipsilateral breast cancer recurrence was reported in 3 patients $(6 \%)$. Two of them occurred outside the treated volume. 5- and 7-year rates of actuarial local control 
were $96 \%$ and $96 \%$, respectively, overall survival $88 \%$ and $85 \%$, disease free survival $88 \%$ and $88 \%$, respectively. Independent cosmetic scoring showed good or excellent result in $56 \%$ of patients. Authors concluded that local outcome was favorable and very similar to other published studies of accelerated partial breast irradiation. Their long time cosmetic results were lower than other published results.

PDR-BT is often used as a palliative irradiation of local recurrences and breast cancer metastasis. This technique is chosen frequently because of higher therapeutic ratio (sparing of healthy tissues) and possibility of fast delivery of higher radiation dose comparing to EBRT. Fritz et al. [27] referred 52 patients suffering from cutaneous metastases at the thoracic wall treated with 54 fields and total doses of 38 to 50 Gy (median 42 Gy) applying 2 PDR courses with a pause of 4 to 5 weeks. Pulses of 1 Gy reference dose at the skin surface were applied at a rate of 1 pulse every 1.2 hours ( 0.8 Gy per hour). The median follow-up was 16 months (range 7.1 to 46.2 months). Local control was achieved in 40 out of 48 fields ( $83 \%$ ) or 41 of 46 patients $(89 \%)$, respectively. Moist desquamation occurred in $52 \%$ of the patients. Late reactions were evaluated after 6 months of minimum follow-up. Thirty-two fields had been previously irradiated with external beam therapy with doses of 40 to $60 \mathrm{~Gy}$. Regardless of whether the skin was pre-irradiated or not, all patients surviving long enough developed telangiectasia within 2 years after PDR irradiation. In pre-irradiated patients $(n=32)$ skin contractures and/or skin necrosis occurred in $12 \%$ each. In newly irradiated patients $(n=14)$ no contractures or skin necrosis were observed [27]. Harms et al. [28] reported in a retrospective study on the effect and toxicity of chest wall re-irradiation using PDR-BT. Between 1993 and 1999, a total of 58 patients were treated. All presented patients experienced locally recurrent breast cancer ( 31 patients had concomitant distant metastases) after mastectomy and had previously completed course of radiation therapy (median, $54 \mathrm{~Gy}$; range, 36-70). Indication for re-irradiation was a progressive macroscopic skin recurrence in 30 cases and incomplete surgical resection in 28 patients. Standard treatment consisted of a split course with two fractions of 20 Gy (interval, 31 days), 0.5-1 Gy/pulse/hourly. The median follow-up was 18 months (range, 7-84). The actuarial 1-, 2- and 3-year local recurrence-free survival rates in patients treated for macroscopic disease (microscopic disease in parenthesis) were $89 \%$ (96\%), 81\% (85\%), and $75 \%(71 \%)$. Local control was obtained in $24 / 30$ $(22 / 28)$ patients. Twenty-nine of the 34 patients $(85 \%)$ who deceased during follow-up were locally controlled. 9/58 patients experienced Grade III acute toxicity, 35/58 patients Grade III (29/58 telangiectasia, 6/58 contracture), and 4/58 Grade IV late toxicity (RTOG/EORTC). Authors concluded that re-irradiation of the chest wall using PDR brachytherapy molds is effective and provides high local control rate with acceptable toxicity [28].

\section{Prostate cancer}

Izard et al. [29] presented preliminary outcomes of PDRBT, EBRT and hormonotherapy for prostate cancer.
The number of 165 consecutive patients with stage T1-T3, N0, M0 prostate cancer were analyzed. Hormones application were used in every patient. Median follow-up was 36 months. Risk groups were low (either Stage $\leq$ T2a, \pm Gleason score $\leq 6, \pm$ Prostate-Specific Antigen [PSA] level $\leq 10 \mathrm{ng} / \mathrm{mL}$ ); intermediate (either stage T2b,c, \pm Gleason score 7, \pm PSA $10-20 \mathrm{ng} / \mathrm{mL}$ ); and high (either stage T3, \pm Gleason score $8-10, \pm$ PSA $>20 \mathrm{ng} / \mathrm{mL}$ ). At 3 years, Radiotherapy Oncology Group (RTOG) Grade III and IV genito-urinary toxicity was $4 \%$ and $1.4 \%$; RTOG Grade III and IV gastro-intestinal toxicity was $2.6 \%$ and $0 \%$, respectively. Erectile preservation was $61 \%$. OS was $93 \%$ (154 of 165) and cause-specific survival was 98\% (162 of 165). At 3 years, disease free survival (DFS) was $93 \%$ (153 of 165). DFS for low-, intermediate-, and high-risk groups was $100 \%, 97 \%$, and $81 \%$, respectively ( $p=0.0003$ ). The nadir plus $2 \mathrm{ng} / \mathrm{mL}$ definition $(p=0.0007)$ best predicted clinical failure, having the lowest false-positive rate (3 of 165). The nadir plus $2 \mathrm{ng} / \mathrm{mL}$ PSA-progressionfree survival (PSA-PFS) rate was $100 \%, 95 \%$, and $87 \%$ for the low-, intermediate, and high-risk groups, respectively. Overall ASTRO PSA-PFS rate was $88 \%$. Authors concluded that PDR-BT plus EBRT is effective in treating localized prostate cancer, with acceptable toxicity. However, median 5-year PSA-PFS follow-up is required before providing a solid recommendation [29].

\section{Head and neck cancer}

De Pree et al. [4] in a retrospective study analyzed the feasibility, toxicity, and preliminary oncologic results in a series of 17 patients treated with interstitial PDR-BT. Tumor localization was as follows: 6 patients - floor of the mouth, 1 - oropharynx, 3 - tongue, 4 - lip, 3 - metastases in lymph nodes. Median total dose was $41.1 \mathrm{~Gy}$, pulse dose ranged from 0.4 to $1 \mathrm{~Gy}$. OFS was $70.6 \%$ in 18 months of follow-up. Early complications included mucositis $(n=4)$, xerostomia $(n=1)$ and infection $(n=3)$. In 1 case necrosis was observed (patient with lymph node recurrence). Levendag et al. [30] reported 38 patients with tonsillar fossa and/or soft palate tumors treated with brachytherapy, 19 of them with PDR-BT. PDR consisted of pulses of $\leq 2$ Gy given 4-8 times daily. 11 patients had T3-4 tumors. Furthermore EBRT was the addend and total summarized median dose was 66 Gy (55-73 Gy). The results in these group were compared to 72 patients treated with EBRT alone (median dose $70 \mathrm{~Gy}$ ). Excellent locoregional control was achieved and only in $13 \%$ of patients $(5 / 38)$ during 3 -years follow-up local recurrence occurred. Three of them were successfully treated with the "salvage surgery". Neither BT scheme or tumor site influenced results. This results contrast with the EBRT-only group where 39\% of patients (28/72) developed local failure [30]. Strnad et al. [31] evaluated the relative incidence of toxicity and local control in patients with head and neck malignancies who underwent interstitial PDR-BT. 47 patients were reported. 40 patients received brachytherapy as a part of their curative treatment regimen, and 7 patients were implanted for palliative purposes and excluded from the analysis of therapy efficacy. 24 patients recieved interstitial brachytherapy procedures alone with total dose of $50 \mathrm{~Gy}$; in 
23 patients, PDR-BT procedures were performed with total dose of $24 \mathrm{~Gy}$ in combination with EBRT. Pulse dose of 0.5 Gy was prescribed to 38/47 patients and 0.7 Gy - to 9/47 patients, hourly, $24 \mathrm{~h}$ a day. After a median followup of 12 months (5-18 months), soft tissue necrosis was observed in one patient and bone necrosis in another case. Permanent locoregional tumor control was achieved in 37 of 40 patients. No distant metastases were observed. Authors concluded that PDR-BT brachytherapy with $0.5-0.7 \mathrm{~Gy} / \mathrm{h}$ is a safe therapy. Their preliminary results suggested that PDR-BT of head and neck cancer is comparable with LDR-BT [31].

\section{Anal and rectal cancer}

Roed et al. [32] treated 17 patients with anal carcinoma using PDR-BT. The treatment consisted of three-field external irradiation of 46 Gy in 23 fractions with five fractions a week to the anal canal and regional pelvic lymph nodes. PDR brachytherapy of 25.2 Gy was applied to the tumor space with 42 pulses of $0.6 \mathrm{~Gy} /$ hourly, within seven to 33 days after completion of EBRT. One local recurrence (LR) has been noted 13 months after brachytherapy. Another failure was observed in patient with liver metastasis and 3 LR occurred in inguinal lymph nodes. Necrosis has been noted in 13 patients within 1-49 weeks (median 16 weeks) after implantation. 8 of this patients required colostomy. Final conclusion: the treatment is highly effective, but with substantial toxicity. De Pree et al. [4] described 3 patients with rectal cancer, 3 with anal cancer and one with a recurrence. Pulse dose ranged from 0.4 to $1 \mathrm{~Gy}$, median total dose was $20 \mathrm{~Gy}$. The most important complications were: fistula rectumvaginalis $(n=1)$, fibrosis perianalis $(n=1)$, chronic mucositis $(n=2)$, fibrosis in sigmoid $(n=1)$, diarrhea $(n=2)$. Gerard et al. [33] presented a series of 19 patients with anal cancer treated between 1995 and 1997. All patients were treated with curative intent with EBRT (44$50 \mathrm{~Gy}$ ) and one or two cycles of concomitant fluorouracil/ cisplatinum. After a gap of 2-3 weeks, PDR interstitial brachytherapy was performed with a rigid needles technique. The dose ranged between 10-25 Gy. After 2 years follow-up all patients are alive. No severe Grade 3-4 toxicity was encountered. One local relapse and one metastasis were seen in two distinct patients. Authors concluded that PDR-BT is an attractive alternative to LDRBT [33]. Bruna et al. [34] evaluated the results of PDR-BT in squamous cell anal canal carcinoma (SCACC). 71 patients with SCACC were treated with PDR-BT. The TNM classification was: 14 T1, 41 T2, 15 T3 and 1 T4, 52 N0, 13 N1, 3 N2 and 3 N3. Treatment started with EBRT to the posterior pelvis (mean dose: $45.5 \mathrm{~Gy}$ ). 47 patients received chemotherapy (neoadjuvant/concomitant or both). After an interval of 2-6 weeks, PDR-BT was performed. The mean dose was 17.8 Gy to the $85 \%$ reference isodose of Paris system. With a median followup of 28.5 months, 2-year actuarial overall survival was $90 \% .14$ relapses occurred (4 distant, 3 regional and 7 local). 10 patients developed Grade III complication (Lent Soma scale) and 2 cases with Grade IV complication (colostomy or abdominal perineal resection for necrosis) were noted.
PDR appeared to be an effective treatment for SCACC and it was capable of reproducing the results usually observed with continuous LDR [34].

\section{Esophageal cancer}

In one of the first publicized results regarding PDR-BT, the outcome of 3 patients with esophageal cancer were presented [35]. 2 patients were irradiated with curative intent after EBRT (56 Gy) and one with recurrence after EBRT. PDR-BT total dose was 16 Gy with reference point $1 \mathrm{~cm}$ in 40 hours (40 pulses). The improvement of clinical status was observed in the next few months. The greatest technical problem was fixing of the applicator during many hours of treatment. Harms et al. [36] evaluated the feasibility, effects, and toxicity of PDR-BT for re-irradiation of oesophageal carcinoma. A total of 16 patients (median age 67 years) with inoperable recurrences from oesophageal cancer after primary radio-/chemotherapy (median 50 Gy) were re-irradiated using PDR-BT (192Ir, $37 \mathrm{GBq})$. The treatment was carried out on an outpatient basis with a weekly 5 Gy daytime schedule (0.5 Gy, pulse hourly, total dose 15-20 Gy) application. The dose was prescribed $10 \mathrm{~mm}$ from the mid-dwell position and encompassed the clipped tumor extension with $2 \mathrm{~cm}$ margins. The use of clips for delineation of tumor extent and catheter movement during irradiations was evaluated. All 61 PDR treatments were applied safely. The median catheter movement was $5 \mathrm{~mm}$, range $2-12 \mathrm{~mm}$. After median follow-up of 8 months, 3 patients experienced complete remission and in five cases partial remission was noted. The median Grade II (RTOG/EORTC) dysphagiafree survival was 17 months. 7 patients experienced Grade I, 5 Grade II, and 1 Grade III late toxicity. 3 patients with uncontrolled locoregional disease showed Grade IV complications: oesophagotracheal fistulae $(n=2)$, fatal arterial bleeding $(n=1)$. Daytime PDR-BT proved to be feasible and provided effective palliation, however the toxicity continued to be a major problem. Thus, the total dose should be restricted to < 15 Gy as regards to such palliative circumstances [36].

\section{Bile duct cancer}

Skowronek et al. [37] analyzed the feasibility of intraluminal palliative PDR-BT in the treatment of locally advanced bile duct and pancreas cancer. 48 patients with advanced bile duct or pancreas cancer, disqualified from surgery or radical EBRT, were treated with trans-hepatic technique and intraluminal PDR-BT: 29 patients with bile duct cancer and 19 - pancreas cancer. 44 patients were treated exclusively with PDR-BT, 4 with PDR-BT, concomitant chemotherapy or surgery. Percutaneous transhepatic technique was used to implant the catheter into bile ducts. Most of patients (38/48, 79\%) received 25 pulses of $0.8 \mathrm{~Gy}$ hourly with the total dose of $20 \mathrm{~Gy}$. In 8 cases PDR was repeated after one week. In all cases, the transhepatic technique allowed insertion of BT catheter into bile duct and safe application of PDR-BT. In 19 out of 29 (65.5\%) of bile duct cancer cases and in 10 out of 19 (52.6\%) of pancreas cancer patients clinical improvement (decrease 
of jaundice) was noted in first control after 4 weeks. Median overall survival time (OS) for bile ducts cancer patients was 11.2 months and for pancreas cancer patients - 5.2 months. Authors concluded that the use of PDR-BT was feasible and had a low early complication rate, and a new percutaneous trans-hepatic technique allowed the treatment (insertion of catheter, PDR brachytherapy) to be performed in one day. In most cases a satisfied palliative effect was achieved, however it was more apparent in bile duct cancer patients then in pancreas cancer patients.

\section{Conclusions}

PDR-BT offers several advantages over conventional LDR-BT: 1 . The distribution of radiation dose can be more easily controlled and tailored permitting the following improvements: 1.a. more precise application (then LDR) of the prescribed dose to the treatment volume, 1.b. better reproducibility of treatment plans, 1.c. greater flexibility in changing the dose distribution through the course of treatment if necessary. 2. Improved radiation safety for clinical and physics staff. 3 . Only one source to be replaced every three months. 4 . All brachytherapy techniques such as intracavitary, interstitial, intraoperative, intraluminal are feasible with one machine. Compared to HDR-BT, PDR offers similar quality of treatment, similar treatment procedure and technical verification, improved radiation safety for clinical and physics staff. Requirements for shielding are less stringent - an accelerator type bunker is not necessary. We note theoretical radiobiological advantage - PDR-BT allows some repair in late-reacting normal tissue due to intervals between pulses. New generation brachytherapy units (e.g. Microselectrons HDR/PDR from Nucletron ${ }^{\circledR}$ ) permits to choose adequate source activity according to clinical situation. PDR requires more involvement of the staff, but in certain clinical situations improves the therapeutic index which is significant especially for patients treated with radical therapy.

\section{References}

1. Nag S. High Dose Rate Brachytherapy - a Textbook. Futura Publishing Company Inc., Armonk, New York 1994.

2. Mould RF, Battermann JJ, Martinez AA. Brachytherapy - from Radium to Optimization. Nucleotron B.V. 1994.

3. Pierquin B, Marinello G. A Practical Manual of Brachytherapy. Medical Physics Publishing, Madison, Wisconsin 1996.

4. de Pree Ch, Popowski Y, Weber D. Feasibility and tolerance of Pulsed Dose Rate interstitial brachytherapy. Int J Radiat Oncol Biol Phys 1999; 43: 971-976.

5. Pulsed Brachytherapy Workshop. A Review. Curr Oncol 1997; 4, suppl. 1.

6. Speiser BL, Mould RF. Brachytherapy for the $21^{\text {th }}$ Century. Nucleotron B.V. 1999.

7. van Eijkeren $\mathrm{M}$, Thienpont $\mathrm{M}$, Boterberg T etal. Implementation, uptime, and safety of a pulsed dose rate afterloading machine. Int J Radiat Oncol Biol Phys 1996; 36: 1233-1237.

8. Brenner DJ, Hall EJ. Conditions for the equivalence of continuous to pulsed dose rate brachytherapy. Int J Radiat Oncol Biol Phys 1991; 20: 180-190.

9. Fowler JF, Mount M. Pulsed brachytherapy: the conditions for no significant loss of therapeutic ratio compared with traditional low dose rate brachytherapy. Int J Radiat Oncol Biol Phys 1992; 23: 661-669.
10. Fowler JF, Van Limbergen EF. Biological effect of pulsed dose rate brachytherapy with stepping sources if short half-times of repair are present in tissues. Int J Radiat Oncol Biol Phys 1997; 37: 877-883.

11. Brenner DJ, Hall EJ, Randers-Pehrson G et al. Quantitative comparisons of continuous and pulsed low dose rate regimens in a model late-effect system. Int J Radiat Oncol Biol Phys 1996; 34: 905-910.

12. Brenner DJ, Schiff PB, Huang Y et al. Pulsed-dose-rate brachytherapy: design of convenient (daytime-only) schedules. Int J Radiat Oncol Biol Phys 1997; 39: 809-815.

13. Visser AG, van den Aardweg GJMJ, Levendag PC. Pulsed dose rate and fractionated high dose rate brachytherapy: choice of brachytherapy schedules to replace low dose rate treatments. Int J Radiat Oncol Biol Phys 1996; 34: 497-505.

14. Skowronek J, Piotrowski T, Zwierzchowski G. PDR brachytherapy - describing of a method and a review of clinical applications. Rep Pract Oncol Radioth 2001; 4: 197-202.

15. Skowronek J, Piotrowski T. Brachyterapia nowotworów metodą Pulsed Dose Rate - opis metody oraz przegląd wskazań do leczenia. Przegl Lek 2002; 59: 31-36 [Polish].

16. Skowronek J, Roszak A, Cikowska-Woźniak E. Brachyterapia nowotworów ginekologicznych metodą pulsacyjną (Pulsed Dose Rate) - opis metody i zastosowanie kliniczne. Gin Pol 2005; 8: 661-670 [Polish].

17. Klimek M, Urbański K, Góra E et al. Rak endometrium wstępna ocena tolerancji pooperacyjnej pulsacyjnej brachyterapii. Rep Pract Oncol Radioth 2000; 5: 10 [Polish].

18. Serkies K, Badzio A, Sawicki T et al. PDR brachytherapy: a report on one-year clinical experience at the Medical University of Gdańsk. Rep Pract Oncol Radioth 2001; 3: 135-140.

19. Swift PS, Purser P, Roberts LW et al. Pulsed low dose rate brachytherapy for pelvic malignancies. Int J Radiat Oncol Biol Phys 1997; 37: 811-817.

20. Rogers CL, Freel JH, Speiser BL. Pulsed low dose rate brachytherapy for uterine cervix carcinoma. Int J Radiat Oncol Biol Phys 1999; 43: 95-100.

21. Jensen PT, Roed H, Engelholm SA et al. Pulsed dose rate (PDR) brachytherapy as salvage treatment of locally advanced or recurrent gynecologic cancer. Int J Radiat Oncol Biol Phys 1998; 42: 1041-1047.

22. Skowronek J. Brachyterapia PDR (pulsacyjna) w leczeniu raka piersi. Wspólcz Onkol 2007; 2: 72-81 [Polish].

23. Fritz P, Berns C, Anton HW et al. PDR brachytherapy with flexible implants for interstitial boost after breast-conserving surgery and external beam radiation therapy. Radiother Oncol 1997; 45: 23-32.

24. Harms W, Krempien R, Hensley FW et al. 5-Year Results of Pulsed Dose Rate Brachytherapy Applied as a Boost after Breast-Conserving Therapy in Patients at High Risk for Local Recurrence from Breast Cancer. Strahlenther Onkol 2002; 178: 607-614.

25. Mangold CA, Rijnders A, Georg D et al. Quality control in interstitial brachytherapy of the breast using pulsed dose rate: treatment planning and dose delivery with an Ir-192 afterloading system. Radiother Oncol 2001; 58: 43-51.

26. Johansson B, Karlsson L, Liljegren G et al. Pulsed dose rate brachytherapy as the sole adjuvant radiotherapy after breastconserving surgery of T1-T2 breast cancer: first long time results from a clinical study. Radioth Oncol 2009; 90: 30-35.

27. Fritz P, Hensley FW, Berns C et al. Long-term results of pulsed irradiation of skin metastases from breast cancer. Effectiveness and squeal. Strahlenther Onkol 2000; 176: 368-376.

28. Harms W, Krempien R, Hensley FW et al. Results of chest wall re-irradiation using pulsed-dose-rate (PDR) brachytherapy molds for breast cancer local recurrences. Int I Radiat Oncol Biol Phys 2001; 49: 205-210. 
29. Izard MA, Haddad RL, Fogarty GB et al. Six year experience of external beam radiotherapy, brachytherapy boost with a 1Ci 192Ir source, and neoadjuvant hormonal manipulation for prostate cancer. Int J Radiat Oncol Biol Phys 2006; 66: 38-47.

30. Levendag PC, Schmitz PIM, Jansen PP et al. Fractionated highdose-rate and pulsed-dose-rate brachytherapy: First clinical experience in squamous cell carcinoma of the tonsillar fossa and soft palate. Int J Radiat Biol Phys 1997; 38: 497-506.

31. Strnad V, Lotter M, Grabenbauer G et al. Early results of pulsed-dose-rate interstitial brachytherapy for head and neck malignancies after limited surgery. Int J Radiat Oncol Biol Phys 2000; 46: 27-30.

32. Roed H, Engelholm SA, Svendsen LB et al. Pulsed dose rate (PDR) brachytherapy of anal carcinoma. Radiother Oncol 1996; 41: 131-134.

33. Gerard J-P, Mauro F, Thomas L et al. Treatment of squamous cell anal canal carcinoma with pulsed dose rate brachytherapy. Feasibility study of a French cooperative group. Radioth Oncol 1999; 51: 129-131.

34. Bruna A, Gastelblum P, Thomas L et al. Treatment of squamous cell anal canal carcinoma (SCACC) with pulsed dose rate brachytherapy: a retrospective study. Radioth Oncol 2006; 79: 75-79.

35. Activity - Nucleotron Special Report. 1994, Nr 5.

36. Harms W, Krempien R, Grehn C et al. Daytime pulsed dose rate brachytherapy as a new treatment option for previously irradiated patients with recurrent oesophageal cancer. BJR 2005; 78: 236-241.

37. Skowronek J, Sowier A, Skrzywanek P. Trans-hepatic technique and intraluminal Pulsed Dose Rate (PDR) brachytherapy in treatment of locally advanced bile ducts and pancreas cancer. J Contemp Brachyther 2009; 1: 97-104. 